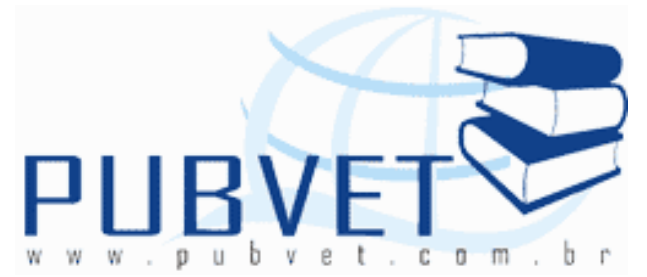

PUBVET, Publicações em Medicina Veterinária e Zootecnia.

\title{
Topografia do cone medular de Ouriço-cacheiro (Coendou prehensilis, Linnaeus, 1758) (Rodentia)
}

André Luiz Quagliatto Santos ${ }^{1}$, Saulo Fernandes Mano de Carvalho ${ }^{1}$, Lorena Tannús Menezes ${ }^{2}$, Liliane Rangel Nascimento ${ }^{2}$, Arthur Paulino Sanzo Kaminishi ${ }^{2}$, Tatiana Grillo Leonardo ${ }^{2}$

Laboratório de Ensino e Pesquisas em Animais Silvestres - LAPAS, FAMEV/UFU, e-mail: quagliatto@famev.ufu.br 1. Docentes. 2. Mestrandos.

\section{Resumo}

A anestesia caudal foi proposta pela primeira vez em 1926 e diversas técnicas foram desenvolvidas para anestesiar os nervos espinhais lombares e sacrais. Este estudo determinou a topografia do cone medular de Coendou prehensilis, utilizando seis espécimes adultos, vindos do Zoológico Parque do Sabiá Uberlândia-MG. No LAPAS estes foram fixados em solução aquosa de formol à $10 \%$. Foi feita incisão de pele, os músculos da coluna vertebral foram retirados, os arcos vertebrais foram seccionados para visualização da medula espinhal e seus envoltórios. Em todos os espécimes o cone medular iniciou-se na quarta vértebra lombar (L4) e terminou na segunda vértebra sacral (S2), o seu comprimento variou de $5,9 \mathrm{~cm}$ to $4,7 \mathrm{~cm}$. Conclui-se que o melhor local para anestesia epidural do membro pélvico no Coendou prehensilis é entre as vértebras L4 e S2.

Palavras-chave: anatomia, medula espinhal, roedores, anestesia. 


\title{
Topography of the medullary cone of Brazilian porcupine (Coendou prehensilis, Linnaeus, 1758) (Rodentia)
}

\begin{abstract}
The first proposed of caudal anesthesia was in 1926 and many techniques were developed to anesthetize the lumbar and sacral spinal nerves. This study determined the medullary cone topography of the Coendou prehensilis, using six adults from the Sabiá Park Zoo, Uberlândia-MG. The material was fixed in formaldehyde solution $10 \%$ in the LAPAS. The skin incision was done, then the spine muscles were removed and the vertebral arches were cut to visualize the spinal cord and the surrounding structures. The medullary cone in all the specimens began in the fourth lumbar vertebra (L4) and finished in the second sacral vertebra (S2), and the length changed to $5.9 \mathrm{~cm}$ to $4.7 \mathrm{~cm}$. This study concluded that the best place for epidural anesthesia of the pelvic limb in the Coendou prehensilis is between the L4 and S2 vertebrae.
\end{abstract}

Keywords: anatomy, spinal cord, rodents, anesthesia.

\section{INTRODUÇÃO}

O conhecimento morfológico das relações entre a medula e a coluna vertebral é de importância para os métodos veterinários e biólogos, permitindo o conhecimento comparado a outras espécies silvestres e domesticas principalmente no emprego de técnicas anestésicas e dentre elas a anestesia espinhal (CARVALHO, 2004).

Durante o desenvolvimento embrionário, os segmentos da medula espinhal e as vértebras estão locadas em um mesmo nível topográfico (SINOWATZ,1991). Em gatos adultos, contudo, a coluna vertebral tem um crescimento maior do que a medula espinhal e a cauda equina estendem-se em grandes extensões (GOLLER, 1966; HOPINKS, 1935; MAIERL et al., 1997; THOMAS e COMBS, 1962). 
A anestesia regional epidural é uma técnica anestésica muito utilizada, e valiosa, em determinados procedimentos em Medicina Veterinária.

Segundo Câmara Filho et al. (2000), gatos ainda são vistos por alguns como pequenos cães, em detrimento de inúmeras diferenças morfológicas, bioquímicas e fisiológicas, dentre outras, que exigem, cada vez mais, um maior grau de especialização dos profissionais que lidam com estas espécies.

Teoricamente, pode-se aplicar a solução anestésica no espaço epidural de qualquer ponto do canal vertebral, tendo em vista que este espaço existe ao longo de toda coluna, onde houver dura-máter. O local de aplicação do anestésico varia conforme a espécie animal, de acordo com o local de término da medula espinhal. Para Hilbery (1992); utiliza-se sítios caudais ao cone medular, o que torna a técnica de aplicação mais segura evitando-se, assim, lesões a medula espinhal.

Em cães o cone medular (limite caudal da medula espinhal) situa-se entre L6 e L7, e, por conseguinte, o sítio para a anestesia epidural é o espaço lombossacro, o que também é recomendado pela maioria dos autores para os gatos (BHON, 1981; HANDERSON, 1977).

Com isto, a eficiência, a segurança e a rapidez para utilização prática dos métodos de anestesia epidural, devem-se ao conhecimento da anatomia da região envolvida (HOPKINS, 1935).

O objetivo deste estudo foi oferecer informações sobre a localização do cone medular em relação às vértebras lombares e sacrais em Coendou prehensilis.

\section{MATERIAL E MÉTODO}

Utilizaram-se seis ouriços-cacheiros Coendou prehensilis, adultos, sendo cinco machos e uma fêmea, provenientes de morte natural no Zoológico Parque do Sabiá, Uberlândia, MG. Estes foram fixados em solução de formol a $10 \%$, em seguida, efetuou-se a retirada da pele e musculatura da região 
dorsal da coluna vertebral, em seguida seccionaram-se os arcos vertebrais, expondo-se a medula espinhal e seus envoltórios.

Individualizou-se o cone medular identificando a sua base e o seu ápice em relação às vértebras lombares e sacrais, mensurando com auxílio de um paquímetro (Starrett/125B). O comprimento corporal do animal foi medido, isto se deu do espaço interarcual occipito-atlântico até o espaço entre a última vértebra sacral e a primeira vértebra caudal.

\section{RESULTADOS}

Os resultados foram: o primeiro Coendou prehensilis macho apresentou $3,6 \mathrm{~cm}$ de comprimento corporal, o seu cone medular iniciou-se na quarta vértebra lombar (L4) e terminou na segunda vértebra sacral (S2), o seu comprimento foi de $5,2 \mathrm{~cm}$. O segundo espécime macho apresentou $3,7 \mathrm{~cm}$ de comprimento corporal, o cone medular iniciou na quarta vértebra lombar (L4) e terminou na segunda vértebra sacral (S2), o seu comprimento foi de $5,9 \mathrm{~cm}$. O terceiro espécime macho apresentou $3,8 \mathrm{~cm}$ de comprimento corporal, o seu cone medular iniciou na quarta vértebra lombar e terminou na segunda vértebra sacral (S2), o seu comprimento foi de $4,7 \mathrm{~cm}$. O quarto espécime macho apresentou $3,7 \mathrm{~cm}$ de comprimento corporal, o seu cone medular iniciou-se na quarta vértebra lombar (L4) e terminou na segunda vértebra sacral (S2), o seu comprimento foi de $4,5 \mathrm{~cm}$. O quinto espécime macho apresentou $3,4 \mathrm{~cm}$ de comprimento corporal, o seu cone medular iniciou na quarta vértebra lombar e terminou na segunda vértebra sacral (S2), o seu comprimento foi de $5,4 \mathrm{~cm}$. E a espécime fêmea apresentou $3,1 \mathrm{~cm}$ de comprimento corporal, o seu cone medular iniciou na quarta vértebra lombar (L4) e terminou na segunda vértebra sacral (S2), o seu comprimento foi de $5,5 \mathrm{~cm}$. 


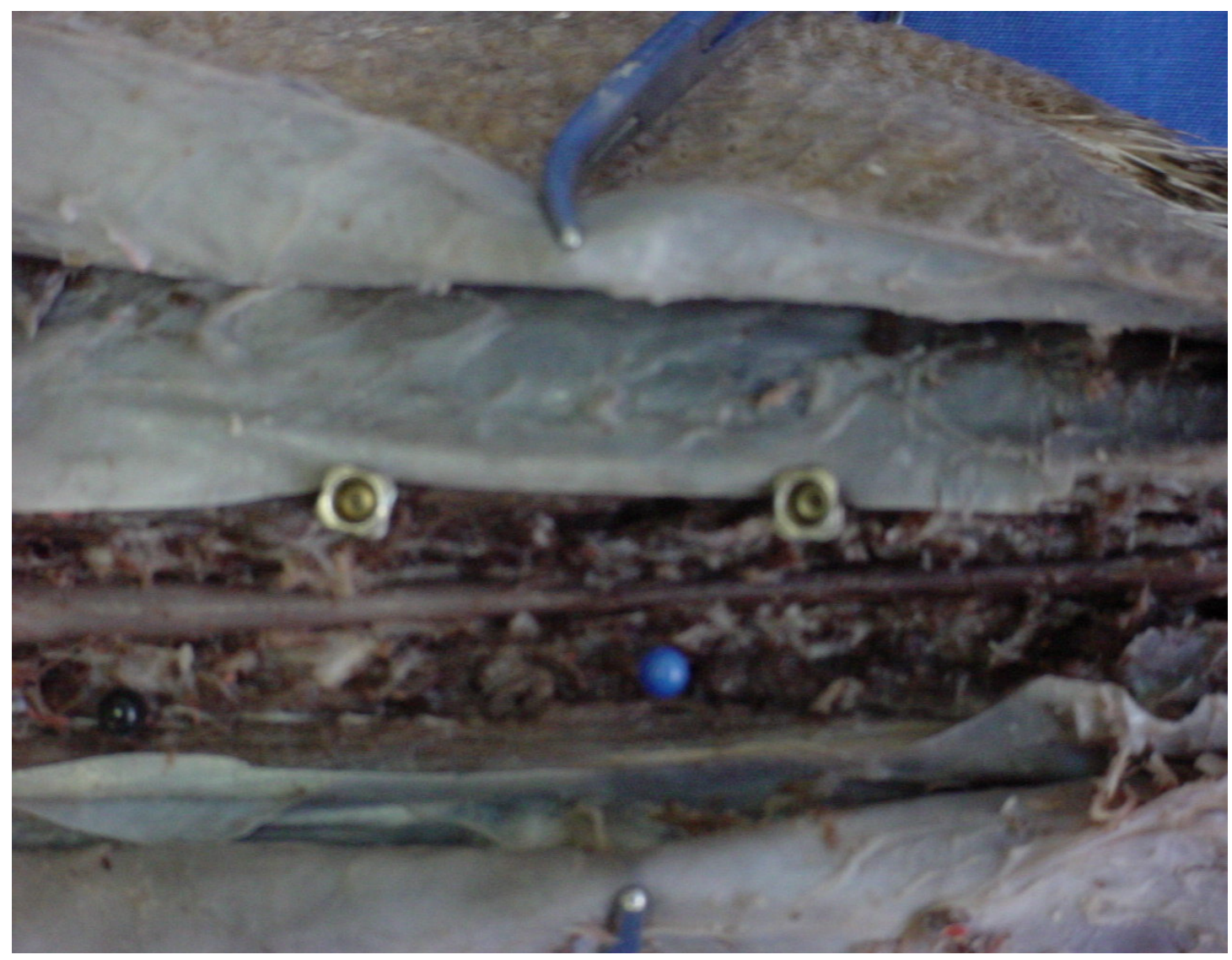

Figura 1: Fotografia da medula espinhal de Coendou prehensilis. As agulhas metálicas delimitam as vértebras sacrais e os alfinetes coloridos delimitam o cone medular.

\section{DISCUSSÃO}

O cone medular alem de ser uma estrutura de suma importância em procedimentos anestésicos veterinários, apresenta terminações que variam em relação a espécie e a idade do animal, ocorrendo entre as últimas vértebras lombares e as primeiras sacrais (BRUNI e ZIMMERL, 1977; DYCE et al., 2004; GETTY, 1986; HOPKINS, 1935; HABEL, 1951; RAO, 1990; RAO et al., 1993; SANTOS et al., 2001; SANTIAGO et al., 1990; SEIFERLE, 1951; SHARMA et al., 1971).

De uma maneira mais detalhada Santos et al. (1999); Santos e Lima (2000); Santos et al. (2001); Santos et al. (1998); Santos et al. (2000) e Silva 
et al. (2009) ofereceram informações à respeito da topografia do cone medular em diversos animais domésticos. Para Dyce et al. (2004) a terminação da medula espinhal varia entre as espécies, ocorrendo em L5 ou L6 nos suínos, em L6 nos ruminantes, L6 ou L7 no cão, S2 no eqüino e, mais variavelmente, entre $\mathrm{L} 6$ e S3 no gato.

Vários autores como Bhon (1981) e Handerson (1977) recomendam o espaço lombossacral como sítio para a anestesia epidural em gatos, seguindo como modelo o sítio na espécie canina. Câmara Filho et al. (1998) citam que o sítio lombossacral não é seguro para gatos, tendo em vista que o cone medular destes animais avança até pontos além de L6 e L7. Nos gatos domésticos, o cone medular atinge o espaço da primeira vértebra sacral. Câmara Filho et al. (2000) encontraram a base do cone medular de gatos domésticos situando-se ao nível da sexta vértebra lombar (L6) e o ápice ao nível da primeira vértebra sacral (S1).

Maierl e Liebich (1998) confirmam estas diferenças, o ápice do cone medular felino situa-se mais caudal à junção lombo sacral. Os resultados indicaram claramente que o sítio de injeção no canal vertebral de gatos é bem diferente do de cães.

Com relação as espécies não domesticas, a topografia do cone medular já foram estudados em humanos (KO et al., 2004; WILLIAMS et al., 1995), nos macacos (CARVALHO-BARROS et al., 2003), nos golfinhos de água doce (FETTUCCIA e SIMÕES-LOPES, 2004); no lobo-guará (Chrysocyon brachyurus), localizando o cone medular entre as vértebras L3 e L6, tendo $6,5 \mathrm{~cm}$ de comprimento (MACHADO et al., 2002); na paca (Agouti paca), o cone medular está entre as vértebras $L 5$ e S1, tendo comprimento de 5,20 a $5,80 \mathrm{~cm}$ (SCAVONE et al., 2007); no lobo marinho (Arctocephalus australis), cuja localização está situada entre as vértebras T5 e T7, caracterizando uma topografia diferente das de outros mamíferos, tendo $4,40 \mathrm{~cm}$ de extensão (MACHADO et al., 2003); na jaguatirica (Leopardus pardalis) um cone medular entre as vértebras $\mathrm{L} 4$ e $\mathrm{S} 3$ e um comprimento total de 8,5cm (BRÍGIDA et al., 2010). 
Carvalho et al. (2003) relataram que no gato-mourisco (Herpailurus yagouaroundi), cone medular se inicia na altura da vértebra L6 e seu ápice está em S2, tendo $5 \mathrm{~cm}$ de comprimento; para Machado et al. (2009b) na ariranha se inicia na vértebra $L 2$ e termina na $L 4$, com 5,5cm de comprimento; Machado e Santos (2008) dizem que no tamanduá-mirim a base do cone é em L1 e o ápice em L5-6, tendo 6,5 a 7,0cm de comprimento; Machado et al. (2009a) no ratão do banhado se inicia na vértebra L4-5 e termina na L3-5, tendo $2 \mathrm{~cm}$ de comprimento; Lima et al. (2010) estudando o veado-catingueiro (Mazama gouazoubira) o cone medular inicia-se entre L2 e L3 e termina em S1 e S2, tem comprimento $4,6 \mathrm{~cm}$; e para Gregores et al. (2010) no quati (Nasua nasua) a base do cone medular está na vértebra L5 e o ápice na vértebra S3, com comprimento entre $5,2 \mathrm{~cm}$ e $5,8 \mathrm{~cm}$.

Comparando o cone medular do ouriço-cacheiro (Coendou prehensilis) estenderam-se da quarta vértebra lombar (L4) até a segunda vértebra sacral (S2), com 5,3cm de comprimento com outro roedor como a paca (Agouti paca) a base em L5 e o ápice em S1 (SCAVONE et al., 2007); observou-se que estes são diferentes, mas possuem um mesmo padrão de distribuição. Sendo assim, para a realização da anestesia epidural no ouriço-cacheiro o sítio de eleição é o sacrococcígeno.

\section{CONCLUSÃO}

O cone medular destes ouriços-cacheiros coendou prehensilis estenderam-se da quarta vértebra lombar (L4) até a segunda vértebra sacral (S2), com $5,3 \mathrm{~cm}$ de comprimento médio, portanto recomenda-se o sítio sacrococcígeno para a realização da anestesia epidural em ouriço-cacheiro.

\section{REFERÊNCIAS}

BOHN, F. K. Device for positioning small animals during anesthesia. Laboratory animals, Inglaterra, v. 15, n. 3, p. 289, Sept. 1981. 
SANTOS, A.L.Q. et al. Topografia do cone medular de Ouriço-cacheiro (Coendou prehensilis, Linnaeus, 1758) (Rodentia). PUBVET, Londrina, V. 5, N. 16, Ed. 163, Art. 1105, 2011.

BRÍGIDA, S. S. S.; BRANCO, E.; PINHEIRO, L. L.; MARTINS, D. M.; ARAÚJO, E. B.; MELUL, R.; LACRETA JR, A. C. C.; MENESES, A. M. C.; SOUZA, A. C. B.; PEREIRA, L. C.; FIORETTO, E. T.; LIMA, A. R. Topografia do cone medular da jaguatirica. Acta Veterinaria Brasilica, Mossoró, v.4, n.1, p.51-54, Out. 2010.

BRUNI A. C.; ZIMMERL U. Anatomia Degli Animali Domestici. Italia: Casa Editrici Dottor Francesco Vallardi, 1977. 736p. v.2.

CÂMARA FILHO, J. A.; RODRIGUES, M.; SILVEIRA, R. Determinação morfológica do cone medular espinhal no espaço da primeira vértebra sacral. Revista do Centro de Ciências Médicas da UFF, Rio de Janeiro, v.2, n.2, p. 55-59, Mar. 1998.

CÂMARA FILHO, J. A.; RAMADINHA, L. S.; RODRIGUES, M. R.; SILVEIRA, R. L.; CALDAS, P. A. Utilização do sítio sacrococcígeo na anestesia epidural em gatos domésticos. Revista Brasileira de Ciência Veterinária, Rio de Janeiro, v.7, n.3, p.175-178, Jan. 2000.

CARVALHO, R. C. Topografia vertebro-medular e anestesia espinhal em Joboti das "Patas Vermelhas" Geochelone carbonária (SPIX, 1824). São Paulo: USP, 2004. 126f. Dissertação (Mestrado em Ciências) Programa de Pós-graduação em Ciências, Faculdade de Medicina Veterinária e Zootecnia, Universidade de São Paulo, 2004.

CARVALHO, S. F. M.; SANTOS, A. L. Q.; AVILA JUNIOR, R. H.; ANDRADE, M. B.; MAGALHÃES, L. M.; MORAES, F. M.; RIBEIRO, P. I. R. Topografia do cone medular em um gato-mourisco, Herpailurus yagouaroundi (Severtow, 1858) (Felidae). Archives of Veterinary Science, Curitiba, v.8, n.2, p.35-38, Fev. 2003.

CARVALHO-BARROS, R. A.; PRADA, I. L. S.; SILVA, Z.; RIBEIRO, A. R.; SILVA, D. C. O. Constituição do plexo lombar do macaco Cebus apella. Brazilian Journal of Veterinary Research and Animal Science, São Paulo, v. 40, n. 5, p. 373- 381, Feb. 2003.

DYCE, K. M., SACK, W. O., WENSING, C. J. G. Tratado de Anatomia Veterinária. 3ed. Rio de Janeiro:Editora Guanabara Koogan, 2004. 813p.

FETTUCCIA, D. C.; SIMÕES-LOPES, P. C. Morfologia da coluna vertebral do boto cinza, Sotalia guianensis (Cetacea, Delphinidae). Biotemas, v.17, n.2, p.125-148, Out. 2004.

GETTY, R. Sisson/Grossman Anatomia dos animais domésticos. 5ed. Rio de Janeiro: Guanabara Koogan, 1986, v.1 e 2.

GOLLER, H. Vergleichende Ruckenmarkstopographie unserer Haustiere. Tierarztliche Umschau, Konstanz, v. 14, n.1, p. 107-110, Mar. 1966.

GREGORES, G. B.; BRANCO, E.; CARVALHO, A. F.; OlIVEIRA, P. C.; FERREIRA, G. J.; CABRAL, R.; FIORETTO, E. T.; MIGLINO, M. A.; CORTOPASSI, S. R. G. Topografia do cone medular do quati. Biotemas, Montes Claros, v.23, n.2, p.35-42, Fev. 2010.

HABEL, R. E. Guide to the Dissection of the Cow. New York: Cornell Cooperative Society, 1951. 375p.

HANDERSON, R. A. Epidural anaesthesia in small animals. Auburn Veterinarian, Auburn, v.34, n.1, p.22-35, Fev. 1977.

HILBERY, A. D. R. Manual de anestesia de los pequeños animales. Zaragoza: Acribia, 1992. 72-75p.

HOPKINS, G. S. The correlation of anatomy and epidural anesthesia in domestic mammals. Cornell Veterinarian, Ithaca, v.25, n.2, p.263-270, Out. 1935. 
SANTOS, A.L.Q. et al. Topografia do cone medular de Ouriço-cacheiro (Coendou prehensilis, Linnaeus, 1758) (Rodentia). PUBVET, Londrina, V. 5, N. 16, Ed. 163, Art. 1105, 2011.

KO, H. Y.; PARK, J. H.; SHIN, Y. B.; BAEK, S. Y. Gross quantitative measurements of spinal cord segments in human. International Spinal Cord Society, United States, v.42, n.1, p.35-40, Mar. 2004.

LIMA, F. C.; SANTOS, A. L. Q.; LIMA, B. C.; VIEIRA, L. G.; HIRANO, L. Q. L. Topographic anatomy of the spinal cord and vertebromedullary relationships in Mazama gouazoubira Fisher, 1814 (Artiodactyla; Cervidae) Acta Scientiarum. Biological Sciences, Maringá, v.32, n.2, p.189-194, Out. 2010.

MACHADO, G. V.; FONSECA, C. C.; NEVES, M. T. D.; PAULA, T. A. R.; BENJAMIN, L. A. Topografia do cone medular no lobo-guará (Chrysocyum brachyurus Illiger, 1815). Revista Brasileira de Ciências Veterinária, Nitoroi, v.9, n. 2, p.107-109, Sept. 2002.

MACHADO, G. V.; LESNAU, G. G.; BIRCK, A. J. Topografia do cone medular no lobo marinho (Arctocephalus australis Zimmermann, 1783). Arquivos de Ciências Veterinárias e Zoologia da Unipar, Umuarana, v.6, n.1, p.11-14, Out. 2003.

MACHADO, G. V.; CAL, J. A.; BIRCK, A. J. Topografia do cone medular no ratão-do-banhado (Myocastor coypus Molina, 1782 - Rodentia: Mammalia). Biotemas, Monte Claros, v.22, n.2, p.117-120, Fev. 2009a

MACHADO, G. V.; LESNAU, G. G.; BIRCK, A. J. Topografia do cone medular no lobo-marinho (Arctocephalus australis Zimmermann, 1803). Arquivos de Ciências Veterinárias e Zoologia da Unipar, Umuarana, v.6, n.1, p.11-14. Nov. 2003.

MACHADO, G. V.; ROSAS, F. C. W.; LAZZARINI, S. M. Topografia do cone medular na ariranha (Pteronura brasiliensis Zimmermann, 1780). Ciência Animal Brasileira, v.10, n.1, p.301305, Jan. 2009b

MACHADO, G. V.; SANTOS, B. S. Topografia do cone medular no tamanduá-mirim (Tamandua tetradactyla Linnaeus, 1758) (Xenarthra: Myrmecophagidae). Archives of Veterinary Science, Curitiba, v.13, n.2, p.172-175, Fev. 2008.

MAIERL, J.; REINDL, S.; KNOSPE, C. Bemerkungen zur Epiduralanasthesie bei der Katze aus anatomischer Sicht. Der PraktischeTierarztl, Hannover, v.25, n.1, p.267-270, Mar. 1997.

MAIERL, J; LIEBICH, H. G. Investigation on the post natal development of the macroscopic proportions and the topographic anatomy of the feline spinal cord. Zentralblatt fur Veterinarmedizin, Reihe C, Anatomia, Histologia, Embryologia, Berlin, v.27, n.1, p.375379, Jan. 1998.

RAO, G. S. Anatomic studies on ovine spinal cord. Anatomischer Anzeiger, Deerfield Beach, v. 171, n.3, p.261-264, Out. 1990.

RAO, G. S.; KALT, D. S.; KOCH, M.; MAJOR, A. A. Anatomical studies on the spinal cord segments of the impala (Alpycerus melanus). Zentralblatt fur Veterinarmedizin, Reihe C, Anatomia, Histologia, Embryologia, Berlin, v. 22, n.1, p. 273-278, Jan. 1993.

SANTIAGO W.; MACHADO, G. V.; VIZIOLI, V. P.; BARRA, P. H. M. Esqueletopia do cone medular em caprinos mestiços. 1990 Belo Horizonte. Anais. Belo Horizonte: IX Congr. Mineiro de Med. Veterinária, 1990, p.107.

SANTOS, A. L. Q.; LIMA, E. M. M ; SANTANA, M. I. S. Lenght of spinal cord and topography of medullar cone in rabbit (Oryctogalus auriculus). Bioscience Journal, Uberlândia, MG, v.15, n.2, p.45-62, Mar. 1999. 
SANTOS, A.L.Q. et al. Topografia do cone medular de Ouriço-cacheiro (Coendou prehensilis, Linnaeus, 1758) (Rodentia). PUBVET, Londrina, V. 5, N. 16, Ed. 163, Art. 1105, 2011.

SANTOS, A. L. Q.; LIMA, E. M. M. Topografia do cone medular em ovinos sem raça definida (Ovies aires-Linnaeus, 1758). ARS Veterinária, Jaboticabal, v.16, n.3, p.154-157, Out. 2000.

SANTOS, A. L. Q.; LIMA, E. M. M.; SANTANA, M. I. S. Comprimento da medula espinhal e topografia do cone medular em coelhos (Oryctolagus cuniculus). 1998, Uberlândia. Anais. Uberlândia: VII Encontro de Iniciação Científica, 1998, p.162.

SANTOS, A. L. Q.; LIMA, E. M. M.; SANTANA, M. I. S. Topografia do cone medular em caprinos da raça Saanen. Arquivos de Ciência Veterinária e Zoologia da UNIPAR, Umuarama, v.4, n.1, p.25-29, Nov. 2001.

SANTOS, A. L. Q.; LIMA, E. M. M. Topografia do cone medular em ovinos sem raça definida (Ovies aires-Linnaeus,1758). Ars Veterinária, Jaboticabal, v.16, n.2, p.154-15, Jan. 2000.

SCAVONE, A. R. F.; GUimarÃES, G. C.; RODRIGUES, V. H. V.; SASAHARA, T. H. C., MACHADO, M. R. F. Topografia do cone medular da paca (Agouti paca, Linnaeus - 1766). Brazilian Journal of Veterinary Research and Animal Science, São Paulo, v.44, n.3, p.53-57, Feb. 2007.

SHARMA, D. N.; RAO, G. S. Topography of spinal cord segments in buffalo (Bubalus bubalis). The Indian Journal Animal Science, Nova Deli, v.41, n.2, p.161-165, Nov. 1971.

SINOWATS, F. Nervensystem. In: Lehrbuch der Embryologie der Haustiere. Berlin: Paul Parey. 1991. p.247-286.

SEIFERLE E. On the topography of the equine and bovine spinal cord. Zeitschrift für Anatomie und Entwicklungsgeschichte, German, v.110, n.3, p.731-784. Jan. 1951.

SILVA, P. H. C.; SILVA, R. M.; LIMA, E. M. M. Topografia do cone medular em gatos sem raça definida. Arquivo Brasileiro de Medicina Veterinária e Zootecnia, Belo Horizonte, v.61, n.3, p.1062-1066, Mar. 2009.

THOMAS, C. E.; COMBS, C. M. Spinal cord segments: A gross structure in the adult cat. American Journal of Anatomy, Philadelphia, v.110, n.2, p.37-47, Nov. 1962.

WILLIANS, P. L.; WARWICK, L.; DYSON, M.; BANNISTER, L. H. Gray anatomia. Rio de Janeiro: Guanabara Koogan, 1995. 